\title{
Avaliação da farmacoterapia prescrita a idosos institucionalizados
}

\author{
Evaluation of pharmacotherapy prescribed to institutionalized elderly \\ Evaluación de la farmacoterapia prescrita para ancianos institucionalizados
}

Francieli Nogueira Smanioto', Maria do Carmo Lourenço Haddad"

' Universidade Estadual de Londrina, Centro de Ciências da Saúde, Curso de Enfermagem (Graduanda). Londrina-PR, Brasil.

"Universidade Estadual de Londrina, Centro de Ciências da Saúde, Departamento de Enfermagem. Londrina-PR, Brasil.

\author{
Submissão: 30-05-2011 Aprovação: 09-06-2013
}

\section{RESUMO}

Estudo transversal, quantitativo, descritivo e exploratório, que objetivou avaliar o perfil e a qualidade da farmacoterapia prescrita a idosos residentes em instituições de longa permanência situadas em Londrina-PR. A população foi constituída por 203 idosos, com predominância do sexo feminino e média de idade de 76,4 anos. Verificou-se que $97,0 \%$ da população fazia uso de algum medicamento. Foram prescritos 151 princípios ativos diferentes, com média de 5,3 princípios ativos prescritos por idoso. Dos indivíduos pesquisados 59,9\% foram submetidos à polifarmácia. Quanto a possíveis interações medicamentosas, foi detectada a ocorrência de 359 , e 58,1\% dos idosos estavam expostos a estes eventos. Verificou-se que foi prescrito ao menos um medicamento de categoria imprópria a 58,1\% dos idosos que faziam uso de medicamentos.

Descritores: Instituição de Longa Permanência para Idosos; Preparações Farmacêuticas; Enfermagem.

\section{ABSTRACT}

Cross-sectional study, quantitative, descriptive and exploratory research, which aimed to evaluate the profile and quality of pharmacotherapy prescribed to elderly residents in long-stay institutions located in Londrina-PR. The population consisted of 203 elderly patients with predominantly female, mean aged 76.4 years. It was found that $97.0 \%$ of the population used one drug. One hundred and fifty one different active ingredients were prescribed, with an average of 5.3 per elderly. From all of the individuals surveyed, 59.9\% were subjected to polypharmacy. It was identified 359 possible drug interactions, and $58.1 \%$ of the elderly were exposed to these events. It was found that was prescribed at least one drug of improper category to $58.1 \%$ of the elderly who were using drugs.

Key words: Nursing Homes; Pharmaceutical Preparations; Nursing.

\section{RESUMEN}

Estudio transversal, cuantitativo, descriptivo y exploratorio, que objetivó evaluar el perfil y la calidad de los medicamentos prescriptos para ancianos residentes en instituciones de longa permanencia de Londrina-PR. La población estuvo constituida por 203 ancianos, con predominio femenino y promedio de edad de 76,4 años. Se encontró que 97,0\% de la población utiliza alguno fármaco. Se prescribieron 151 ingredientes activos diferentes, con una media de 5,3 principios activos por anciano. Entre los participantes, $59,9 \%$ de habían sido sometidos a la polifarmacia. Fueran detectadas 359 posibles interacciones medicamentosas, y $58,1 \%$ de los ancianos estaban a estos eventos. Se encontró que se prescribió al menos una categoría inadecuada de medicamento al 58,1\% de los ancianos en medicación.

Palabras clave: Hogares para Ancianos; Preparaciones Farmacéuticas; Enfermería. 


\section{INTRODUÇÃO}

O avanço da medicina e o uso de medicamentos são importantes fatores para o aumento da longevidade, trazendo inúmeros benefícios aos indivíduos idosos, se utilizados com cautela e responsabilidade. Caso contrário, estes podem piorar as condições de vida desta população pelo grande número de reações adversas aos medicamentos (RAM's) que podem acometê-los ${ }^{(1)}$.

AS RAM's podem ser mais graves e acentuarem-se nos idosos devido às alterações nos processos de absorção, distribuição, metabolismo e excreção dos medicamentos, característicos do envelhecimento(2).

Existem poucos estudos a respeito da farmacoterapia em idosos no Brasil e os artigos encontrados se referem, na sua maioria, a idosos residentes na comunidade ${ }^{(3)}$. Sendo assim torna-se importante a realização de estudos com idosos institucionalizados, que beneficiaria não só a essa população restrita, mas a toda população idosa.

A administração de medicamentos é de responsabilidade da equipe de enfermagem, e trata-se de uma atividade que exige responsabilidade e embasamento científico para ser desenvolvida de forma segura ao paciente ${ }^{(2)}$.

O artigo 11, inciso III, alínea "a" do Decreto Lei $n^{\circ}$ 94.406/87 determina que a administração de medicamentos é uma atribuição do auxiliar de enfermagem, juntamente com o artigo 13 que responsabiliza o enfermeiro pela supervisão, orientação e direção desta atividade ${ }^{(4)}$.

Logo, é necessário que estudos que analisem a qualidade da farmacoterapia prescrita a idosos institucionalizados sejam realizados com o intuito de identificar eventuais erros e contradições para que estes sejam modificados de modo que a prescrição medicamentosa propicie à população idosa uma melhoria da qualidade de vida.

Considerando esse pressuposto teve-se como objetivo avaliar o perfil e a qualidade da farmacoterapia prescrita a idosos residentes em instituições de longa permanência situadas em Londrina-PR.

\section{MATERIAIS E MÉTODOS}

Estudo descritivo e transversal realizado em todas as instituições de longa permanência para idosos (ILPIs), filantrópicas, situadas em Londrina-PR, totalizando 203 idosos distribuídos em quatro ILPIs. Foram incluídos todos os idosos que se encontravam efetivamente residindo nas instituições, sendo excluídos aqueles que durante o período da coleta de dados não tivessem completado 60 anos.

Os dados foram coletados no segundo semestre de 2009, utilizando-se de um instrumento de avaliação contendo dados sócio-econômicos (idade, sexo, escolaridade), tempo de institucionalização, presença de tabagismo, etilismo, deambulação, se já eram aposentados. Estes dados foram obtidos em prontuário e através de informações fornecidas pelos profissionais de saúde responsáveis pelo cuidado dos idosos. Quanto aos medicamentos foram catalogados todos os produtos farmacêuticos prescritos no mês de outubro, obtidos a partir dos registros nos prontuários de todos os residentes, os quais foram solicitados junto aos responsáveis técnicos das respectivas instituições de longa permanência para Idosos (ILPI's).

Foram coletadas as seguintes informações a respeito da farmacoterapia: nome comercial de cada medicamento e principio ativo, ou seja, toda "substância quimicamente ativa responsável pela ação do medicamento representado pelo nome genérico" ${ }^{\prime \prime(5)}$.

Todos os produtos farmacêuticos industrializados e fórmulas magistrais foram incluídos no banco de dados e analisados, exceto os que não puderam ter a sua composição claramente determinada (homeopáticos, fitoterápicos, chás e tinturas). Os princípios ativos encontrados em cada produto foram agrupados por classes terapêuticas, conforme o Anatomical-Therapeutical-Chemical Classification System (ATC). Trata-se de uma ferramenta com a finalidade de melhorar o uso de drogas ${ }^{(6)}$.

Foram descritos os eventos de polifarmácia, considerando o uso de cinco ou mais medicamentos simultaneamente ${ }^{(7)}$. Considerou-se medicamentos impróprios para idosos aqueles descritos pelos critérios canadenses que consiste em uma lista elaborada por meio de um consenso, atualizada posteriormente e dividida em duas categorias: medicamentos ou classes deles que deveriam ser evitados em idosos, independentemente do diagnóstico ou da condição clínica, devido ao alto risco de efeitos colaterais e pela existência de outros fármacos mais seguros; e medicamentos ou classes deles que não devem ser usados em determinadas circunstâncias clínicas ${ }^{(8)}$.

Os resultados foram processados e tabulados no programa Epi Info versão 3.3.2 2005, analisando as variáveis simples por porcentagem e realizadas associações entre variáveis usando razão de prevalência.

O estudo faz parte da pesquisa "Avaliação cognitiva de idosas institucionalizadas", com protocolo na CPG/UEL sob o n 28729/2007, e obteve aprovação do Comitê de Ética em Pesquisa da Universidade Estadual de Londrina, protocolo no SISNEP/ CAAE nº 0245.0.268.000-07.

\section{RESULTADOS E DISCUSSÃO}

A idade dos idosos variou entre 60 e 100 anos e a média e a mediana foram 76,4 e 77 , respectivamente $(D P=8,8)$. A população era predominantemente feminina (Tabela 1). Este quadro pode ser atribuído a fatores como a menor exposição do sexo feminino a determinados riscos, notadamente no trabalho; menor prevalência de tabagismo e uso de álcool; diferenças quanto à atitude em relação a doenças e incapacidades e, por último, maior cobertura da assistência gineco-obstétrica ${ }^{(9)}$.

Observou-se que $84,8 \%$ dos idosos possuíam registro nos prontuários de dados referentes ao estado civil. Estes dados podem indicar que possuir um companheiro diminui a institucionalização do idoso, mas que isso não se aplica ao fato de ter família. Dos idosos investigados 58,8\% possuíam dados disponíveis com relação à escolaridade e desses a maioria $(80,0 \%)$ era analfabeto ou possuía ensino fundamental incompleto. 
Tabela 1 - Caracterização sociodemográfica de idosos residentes em instituições de longa permanência para idosos, filantrópicas. Londrina-PR, 2009.

\begin{tabular}{|c|c|c|c|c|c|c|c|}
\hline & \multicolumn{2}{|c|}{ Masculino } & \multicolumn{2}{|c|}{ Feminino } & \multicolumn{2}{|c|}{ Total } & \multirow{2}{*}{$\begin{array}{c}\% \\
\text { Acumulada }\end{array}$} \\
\hline & N & $\%$ & $\mathrm{~N}$ & $\%$ & $\mathrm{~N}$ & $\%$ & \\
\hline \multicolumn{8}{|l|}{ Faixa etária (anos) } \\
\hline 60 A 69 & 30 & 33,7 & 22 & 19,3 & 52 & 25,6 & 25,6 \\
\hline 70 A 79 & 34 & 38,2 & 46 & 40,4 & 80 & 39,4 & 65,0 \\
\hline 80 A 89 & 20 & 22,5 & 36 & 31,5 & 56 & 27,6 & 92,6 \\
\hline$>90$ & 5 & 5,6 & 10 & 8,8 & 15 & 7,4 & 100,0 \\
\hline \multicolumn{8}{|l|}{ Estado Civil } \\
\hline Solteiro & 47 & 61,8 & 37 & 34,9 & 84 & 46,2 & 46,2 \\
\hline Casado & 6 & 7,9 & 5 & 4,7 & 11 & 6,0 & 52,2 \\
\hline Divorciado/separado & 14 & 8,4 & 9 & 8,5 & 23 & 12,6 & 64,8 \\
\hline Viúvo & 9 & 11,8 & 55 & 51,9 & 64 & 35,2 & 100,0 \\
\hline
\end{tabular}

Em relação ao tempo de institucionalização, 90,2\% dos idosos possuíam este dado disponível no prontuário e, destes, $12,5 \%$ residiam na instituição há menos de um ano, 50,5\% de um a cinco anos, 20,1\% de seis a dez anos e 16,8\% mais que dez anos. O tempo de residência mínimo na ILPI foi de até um ano e o máximo de 46 anos, com uma média de permanência de 6,1 anos. A maioria dos idosos $(93,4 \%)$ era aposentada. $O$ hábito de etilismo foi encontrado em $9,3 \%$ da população e o tabagismo em 21,1\%.

Os achados demonstraram que $97 \%$ dos 203 idosos faziam uso de algum medicamento, dado este superior ao encontrado em instituições de longa permanência para idosos no Nordeste do Brasil $(87,2 \%)^{(3)}$. Desta forma, apenas $3 \%$ não faziam uso de medicamentos.

Neste estudo a média do número de medicamentos prescritos por idoso foi de 5,6, variando de um a 15 produtos, sendo que a maioria fazia uso de mais de cinco medicamentos (Tabela 2). Em estudo realizado em uma instituição asilar do Rio de Janeiro-RJ foi encontrado uma média de 3,3 medicamentos ${ }^{(10)}$.

Tabela 2 - Número de medicamentos prescritos a idosos residentes em instituições de longa permanência para idoso (ILPI) filantrópicas de Londrina - PR, 2009.

\begin{tabular}{lcc}
\hline No de medicamentos & N & \% \\
\hline 1 a 4 & 79 & 40,1 \\
5 a 8 & 92 & 46,7 \\
$>8$ & 26 & 13,2 \\
\hline Total & 197 & 100,0 \\
\hline
\end{tabular}

O número de medicamentos consumidos tende a aumentar com a idade ${ }^{(22)}$. E para cada medicamento utilizado pelo idoso, a chance de internação decorrente de complicações medicamentosas aumenta em $65 \%{ }^{(11)}$.

Entre os 197 medicamentos foram encontrados 151 princípios ativos diferentes prescritos para a população estudada. Considerando cada principio ativo como um evento independente de medicação observou-se um total de 1.071 eventos de medicação realizados para o grupo, o que determinou um número médio de 5,3 princípios ativos prescritos por idoso. A maioria dos medicamentos prescritos era de uso contínuo (Tabela 3).

Tabela 3 - Classificação de medicamentos prescritos a idosos residentes em instituições de longa permanência para idoso (ILPI) filantrópicas, segundo tempo de uso. Londrina-PR, 2009.

\begin{tabular}{lcc}
\hline \multicolumn{1}{l}{ Tempo de uso } & N & $\%$ \\
\hline Contínuo & 999 & 93,3 \\
Se necessário & 65 & 6,1 \\
Tempo determinado & 07 & 0,6 \\
\hline Total & 1.071 & 100,0 \\
\hline
\end{tabular}

Os princípios ativos mais consumidos foram: ácido acetilsalicílico $(5,1 \%)$, hidroclorotiazida $(4,3 \%)$, sinvastatina $(4,3 \%)$, enalapril $(4,0 \%)$, sulfato ferroso $(4,0 \%)$ e captopril $(3,2 \%)$. O ácido acetilsalicílico tem função analgésica e antitrombótica, e tem como principal efeito adverso o desconforto gástrico, principalmente se ingerido sem alimentação prévia; também 
pode agir antagonizando o efeito de anti-hipertensivos, dificultando o controle da pressão arterial $^{(12)}$.

De acordo com a classificação ATC foram encontradas 38 classes de medicamentos. Nesta classificação as substâncias ativas são divididas em diferentes grupos, de acordo com o órgão ou sistema em que atuam e suas propriedades terapêuticas, farmacológicas e químicas ${ }^{(8)}$. Dentre os eventos de medicação, as classes mais prevalentes foram os antipsicóticos $12,1 \%$, os hipolipemiantes $9,6 \%$, os psicoanalépticos $8,5 \%$ e os inibidores do sistema renina-angiotensina 7,6\%.

Com relação às possíveis interações medicamentosas foi detectada a ocorrência de 359 eventos e 55,7\% idosos estavam expostos a estes. Do total de interações medicamentosas $24,8 \%$ foram classificadas como leve, $61,8 \%$ como moderada, e 13,4\% como grave.

Os critérios canadenses de Mcleod nos fornecem uma lista de medicamentos que são impróprios para serem utilizados por idosos, ou seja, cujos danos causados por eles superam os benefícios, possibilitando uma assistência farmacológica mais segura. Como exemplo de medicação imprópria está o uso do fármaco amitriptilina por idosos ${ }^{(8)}$. Baseado nestes critérios, identificou-se neste estudo a prescrição de ao menos um medicamento de categoria imprópria a 58,1\% dos idosos, superior ao encontrado em outro estudo ${ }^{(13)}$. Além disso, os idosos foram expostos a um total de 172 eventos de drogas potencialmente inapropriadas, em que $82,6 \%$ podem determinar efeitos adversos de alta severidade.

Verificou-se que 59,9\% dos idosos que estavam em uso de medicações foram submetidos à polifarmácia. Tais achados encontram-se extremamente superiores aos dados da literatura brasileira ${ }^{(14)}$.

O aumento do consumo de medicamentos é influenciado por fatores socioeconômicos e culturais, neste estudo tem-se como principal fator a prescrição indiscriminada de medicamentos e o aumento do número de patologias decorrentes do envelhecimento, considerando que a única fonte de acesso aos medicamentos dos sujeitos da pesquisa é a prescrição realizada na instituição.

Algumas medidas podem reduzir a polifarmácia, incluindo manter o registro atualizado dos medicamentos, revisando-o a cada consulta; identificar o nome dos medicamentos pelo nome genérico e grupo terapêutico; certificar-se da indicação adequada; ter conhecimento dos efeitos secundários dos medicamentos; conhecer as alterações promovidas pelo envelhecimento e evitar redundâncias farmacológicas ${ }^{(13)}$.

Os critérios anteriormente mencionados são uma importante ferramenta na prática da terapêutica medicamentosa ao idoso institucionalizado(14). Algumas limitações destes critérios dizem respeito ao fato de se tratar de um consenso e não ser um trabalho baseado em evidências; não permitir avaliar fatores como interações medicamentosas, dosagens inapropriadas, tempo de tratamento e erros de prescrição; além de não serem totalmente condizentes com a realidade dos medicamentos disponíveis no Brasil ${ }^{(15)}$.

O uso de medicamentos é de extrema importância à saúde, mas o seu uso indiscriminado e suas consequências geram aumento dos gastos na área da saúde. O aspecto econômico também deve ser observado, considerando que este estudo foi desenvolvido em instituições de longa permanência, filantrópicas, e estas dependem em parte da contribuição de terceiros para manter as suas despesas. Em um estudo realizado em instituições de longa permanência na região sul do Brasil, identificou-se que o gasto com medicamentos em ILPIs filantrópicas equivalia a 4,8\% do orçamento ${ }^{(16)}$.

Estes dados demonstram a importância de uma avaliação adequada no momento da prescrição de medicamentos para idosos e, para isto, é necessária a atuação de uma equipe multidisciplinar, com profissionais capacitados. Portanto, se faz necessário o cumprimento da legislação que exige a permanência de uma equipe de profissionais de saúde nestas instituições, o que permite um acompanhamento adequado e individualizado ${ }^{(17)}$.

A equipe de enfermagem assume um importante papel na terapêutica do paciente, sendo a responsável legal pela administração dos medicamentos prescritos. Tão importante quanto à prescrição médica é a administração dos medicamentos prescritos, função essa exercida pela equipe de enfermagem $^{(18)}$. Por isso, é imprescindível que a equipe de enfermagem e principalmente o enfermeiro tenha conhecimento a respeito da farmacologia de modo que possa identificar possíveis interações medicamentosas, incompatibilidades farmacológicas e reações farmacológicas precocemente minimizando assim os riscos ao cliente ${ }^{(19)}$.

É responsabilidade de o enfermeiro promover ações de educação com a equipe de enfermagem, transformando a prática da administração de medicamentos um ato consciente e seguro para os clientes. Pois, em quase todos os lugares a execução da prescrição médica é cumprida mecanicamente em horários preestabelecidos ${ }^{(20)}$.

\section{CONSIDERAÇÕES FINAIS}

Os resultados deste estudo mostraram que os idosos institucionalizados em locais filantrópicos recebem uma quantidade de medicamentos superiores a de idosos residentes em comunidades e ainda maior se comparados com outras ILPIs. E isso é evidente tanto em relação ao número de medicamentos consumidos, as possíveis interações medicamentosas e no número de medicamentos impróprios para idosos.

Foi possível observar nestas instituições a não permanência de uma equipe multidisciplinar capacitada para o atendimento a esta população. O gerenciamento das instituições é realizado por enfermeiros que na maioria das vezes não possui especialização na área de Gerontologia, sendo um enfermeiro por período, realidade não condizente com a necessidade das instituições, não permitindo assim um acompanhamento e atendimento adequado aos idosos.

O médico faz visitas semanais ou até quinzenais, havendo grande rotatividade desses profissionais, o que não permite um acompanhamento sistemático. Em casos de intercorrências os idosos são levados a hospitais de referência.

Ressalta-se também, a importância do acompanhamento da equipe unidade básica de saúde da área de abrangência, para que o idoso seja assistido de modo integral à saúde.

$\mathrm{O}$ investimento em profissionais capacitados e em métodos 
que permitam uma prescrição de medicamentos adequada é de extrema importância, considerando o elevado custo com internações decorrentes de efeitos adversos e interações medicamentosas, e do gasto desnecessário com medicamentos.

A adoção de instrumentos com critérios que permitam detectar possíveis interações medicamentosas de maneira fácil e precocemente pode ser uma forma de minimizar a ocorrência desses problemas.

A sistematização e padronização dos prontuários fornecem subsídios que permitem detectar possíveis fatores de risco para ocorrência de reações medicamentosas e fazer um acompanhamento individual.

O enfermeiro deve apoderar-se da prática de administração de medicamentos, valorizando esta atividade como uma série de técnicas a serem executadas e como uma atividade reflexiva, pautada em conhecimentos a respeito da terapêutica medicamentosa, anatomia e fisiologia do envelhecimento visando um atendimento de qualidade à população idosa.

\section{REFERÊNCIAS}

1. Passarelli MCG, Jacob Filho W. Reações adversas a medicamentos em idosos: como prevê-las? / Adverse drug reactions in elderly patients: how to predict them? Einstein (São Paulo) 2007;5(3):246-251.

2. Universidade Federal do Ceará. Protocolos de preparo e administração de medicamentos: pulsoterapia e hospital dia. Universidade Federal do Ceará: Fortaleza; 2008.

3. Aguiar PM, Lyra Junior DP, Silva DT, Marques TC. Avaliação da Farmacoterapia de Idosos Residentes em Instituições Asilares no Nordeste do Brasil. Acta Farm Bonaer 2008;27(3):454-59.

4. Conselho Regional de Enfermagem. Principais legislações para o exercício da enfermagem. São Paulo: COREN; 1996.

5. Destruti $A B$, Arone ME,Philippi MLS. Introdução à Farmacologia. São Paulo: SENAC-SP; 1999.

6. WHO Collaborating Centre for Drug Statistics Methodology [homepage na internet]. The Anatomical Therapeutic Chemical Classification System [acesso 21 maio 2013]. Disponível em: http://www.whocc.no/atc/ structure_and_principles/

7. Klarin I, Wimo A, Fastbom J. The association of inappropriate drug use with hospitalization and mortality: a population-based study of the very old. Drugs Aging 2005;22(1)69-82.

8. McLeod PJ, Huang AR, Tamblyn RM, Gayton DC. Defining inappropriate practices in prescribing for elderly people: a national consensus panel. Can Med Assoc J 1997;156(3):385-91.

9. Coelho Filho JM, Ramos LR. Epidemiologia do envelhecimento no Nordeste do Brasil: resultados de inquérito domiciliar. Rev Saúde Pública 1999;33(5):445-53.

10. Gomes MPC, Couto MCV, Pepe VLE, Almeida LM, Delgado PGG, Coutinho ESF. Censo dos pacientes internados em uma instituição asilar no Estado do Rio de Janeiro: dados preliminares. Cad Saúde Pública 2002;18(6):1803-7.

11. Portal da Saúde [homepage na internet]. Fármacos em
Idosos [acesso em: 15 de jun 2010]. Disponível em: http://portal.saude.gov.br/portal/arquivos/multimedia/paginacartilha/docs/farmacosi.pdf.

12. Paz KMR, Rúpolo DJ, Lisboa HCF. Avaliação do uso de medicamentos em idosos. In:Transformação social e sustentabilidade ambiental. Anais do $61^{\circ}$ Congresso Brasileiro de Enfermagem; 2009 dez 7-10; Ceará, Brasil. Ceará: Associação Brasileira de Enfermagem; 2009. p. 3682-3685.

13. Williams CM. Using medications appropriately in older adults. Am Fam Physician 2002;66(10):1917-24.

14. Flores LM, Mengue SS. Uso de medicamentos por idosos em região do sul do Brasil. Rev Saúde Pública 2005;39(6):924-9.

15. Fick DM, Cooper JW, Wade WE, Waller JL, Maclean JR, Beers MH. Updating the Beers criteria for potentially inappropriate medication use in older adults: results of a US consensus panel of experts. Arch Intern Med 2003;163(22):2716-24.

16. Gorzoni ML, Fabbri RMA, Pires SL, Rocha FA. Polifarmácia na primeira avaliação geriátrica. Gerontologia 2004; 12:(81)

17. Agência Nacional de Vigilância Sanitária (Brasil). Resolução RDC $n^{\circ}$. 283, de 26 de setembro de 2005. Aprova o regulamento técnico que define normas de funcionamento para as Instituições de longa permanência para idosos. Diário Oficial da União 27 set 2005;Seção 1.

18. Telles Filho PCP, Cassiani SHB. Administração de medicamentos: aquisição de conhecimentos e habilidades requeridas por um grupo de enfermeiros. Rev Latino-Am Enferm 2004;12(3):533-40.

19. Secoli SR. Polifarmácia: interações e reações adversas no uso de medicamentos por idosos. Rev Bras Enferm 2010;63(1):136-40.

20. Secoli SR. Interações medicamentosas: fundamentos para a prática clínica da enfermagem. Rev Esc Enferm USP 2001;35(1):28-34. 